Received: 24 July 2017

Accepted: 26 October 2017

Published online: 08 November 2017

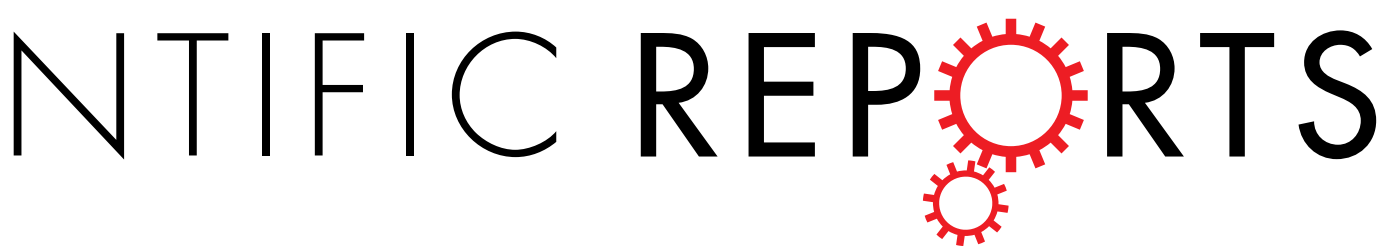

\title{
OPEN Impact of Lactobacillus reuteri colonization on gut microbiota, inflammation, and crying time in infant colic
}

\author{
Monica L. Nation ${ }^{1}$, Eileen M. Dunne ${ }^{1,3}$, Shayne J. Joseph ${ }^{1}$, Fiona K. Mensah ${ }^{1,2,3}$ \\ Valerie Sung ${ }^{1,2,3}$, Catherine Satzke ${ }^{1,3}$ \& Mimi L. K. Tang ${ }^{1,2,3}$
}

Infant colic is a distressing condition of unknown etiology. An aberrant gastrointestinal microbiota has been associated, and Lactobacillus reuteri supplementation has been shown to reduce crying and/or fussing time ('crying time') in some infants with colic. The relationship between $L$. reuteri gut colonization and crying time has not been examined. We investigated the relationship between L. reuteri colonization and fecal microbiota (microbial diversity and Escherichia coli), intestinal inflammation, and crying time in infants with colic, using a subset of 65 infants from the Baby Biotics trial, which randomized healthy term infants aged $<13$ weeks with infant colic to receive probiotic L. reuteri DSM 17938 ( $1 \times 10^{8}$ colony forming units) or placebo daily for 28 days. We observed an overall reduction in median crying time, regardless of $L$. reuteri colonization status $(n=14$ colonized). There were no differences in $E$. coli colonization rates or densities, microbial diversity or intestinal inflammation by $L$. reuteri colonization status. We found that $L$. reuteri density positively correlated with crying time, and $E$. coli density negatively correlated with microbial diversity. As density of $L$. reuteri was associated with increased crying time, $L$. reuteri supplementation may not be an appropriate treatment for all infants with colic.

Infant colic is a distressing condition characterized by crying and/or fussing of unknown cause that affects up to $28 \%$ of infants under three months of age ${ }^{1}$. The etiology of infant colic is unclear and there is no widely accepted or effective treatment ${ }^{2,3}$. Several studies have found differences in gastrointestinal (GI) microbial diversity between infants with colic and healthy infants, suggesting that an aberrant microbial intestinal profile may cause or contribute to infant colic ${ }^{4-6}$. Infants with colic have higher rates and densities of Escherichia coli and other gas-producing coliforms and lower levels of Lactobacillus spp. compared with healthy infants ${ }^{4-7}$, with some aerobic bacterial genera not detected in infants with colic $^{8}$. One study found that infants with colic had lower microbial diversity and elevated fecal calprotectin (a marker of gut inflammation) compared with healthy infants ${ }^{9}$. However, another study found no difference in fecal calprotectin levels between healthy infants and infants with colic $^{10}$.

The finding that infants with colic have an altered intestinal microbiota has led to the investigation of probiotic supplementation for the treatment of this condition, with the aim of promoting a healthy intestinal microbiota and reducing intestinal inflammation. Several clinical trials suggest Lactobacillus reuteri may reduce crying and/ or fussing time (referred to as 'crying time') in some infants with colic ${ }^{11-15}$. In contrast, we recently reported results of a double blind, randomized, placebo-controlled trial evaluating L. reuteri supplementation for the treatment of infant colic in Melbourne, Australia (Baby Biotics trial, Current Controlled Trials ISRCTN95287767 $(25 / 10 / 2010))$ that found no effect on crying time when compared to placebo ${ }^{16}$. However, analysis of fecal samples indicated that less than half of the infants in the probiotic treatment group were colonized with $L$. reuteri at day 28 of the trial, and this low colonization rate may have contributed to the negative trial findings. As probiotics are thought to exert their beneficial effects on the host through transiently colonizing the GI tract, we sought to examine the relationship between L. reuteri colonization and key microbiological, immunological, and clinical characteristics of infant colic. We also investigated the role of $E$. coli, which has been implicated in infant colic $c^{4,7,17}$.

${ }^{1}$ Murdoch Children's Research Institute, Parkville, 3052, Australia. ${ }^{2}$ The Royal Children's Hospital, Parkville, 3052, Australia. ${ }^{3}$ The University of Melbourne, Parkville, 3052, Australia. Correspondence and requests for materials should be addressed to M.L.K.T. (email: mimi.tang@rch.org.au) 


\begin{tabular}{|c|c|c|c|c|c|}
\hline \multirow[b]{2}{*}{ Characteristic } & \multicolumn{2}{|c|}{ Original trial $^{16}$} & \multicolumn{3}{|l|}{ This study } \\
\hline & \begin{tabular}{|l} 
Probiotic \\
$(\mathbf{n}=\mathbf{8 5})$
\end{tabular} & $\begin{array}{l}\text { Placebo } \\
(\mathbf{n}=\mathbf{8 2})\end{array}$ & $\begin{array}{l}\text { Colonized by } L \text {. } \\
\text { reuteri }(\mathrm{n}=14)\end{array}$ & $\begin{array}{l}\text { Not colonized by } L \text {. } \\
\text { reuteri }(\mathrm{n}=51)\end{array}$ & P-value ${ }^{\mathrm{a}}$ \\
\hline Gender Male, $n(\%)$ & $37(44)$ & $48(59)$ & $6(43)$ & $30(59)$ & $0.29^{\mathrm{b}}$ \\
\hline Mode of birth Caesarean section, n (\%) & $35(41)$ & $32(39)$ & $4(29)$ & $20(40)$ & $0.46^{\mathrm{b}}$ \\
\hline Method of feeding Exclusively breastfed, n (\%) & $33(39)$ & $35(43)$ & $6(43)$ & $17(33)$ & $0.51^{\mathrm{b}}$ \\
\hline Family history of allergic disease Yes, n (\%) & $51(60)$ & $50(61)$ & $11(79)$ & $41(80)$ & $0.88^{\mathrm{b}}$ \\
\hline Birth weight (g) Mean \pm SD & $3272 \pm 406$ & $3426 \pm 421$ & $3425 \pm 482$ & $3312 \pm 398$ & $0.57^{\mathrm{c}}$ \\
\hline Infant age at enrolment (weeks) Mean \pm SD & $7.5 \pm 2.9$ & $6.9 \pm 2.5$ & $8.2 \pm 2.8$ & $7.4 \pm 2.7$ & $0.29^{c}$ \\
\hline $\begin{array}{l}\text { Infant crying time at day } 0(\min / \text { day })^{d} \\
\text { Mean } \pm S D\end{array}$ & $327.6 \pm 151.9$ & $329.3 \pm 126.4$ & $337.2 \pm 186.3$ & $344.1 \pm 124.1$ & $0.94^{\mathrm{c}}$ \\
\hline
\end{tabular}

Table 1. Clinical characteristics of the study population. ${ }^{a}$ Differences assessed for infants in the current study (colonized versus not colonized by L. reuteri). ${ }^{\mathrm{b}}$ Chi-Squared test; ${ }^{\mathrm{c}}$ Mann-Whitney U test; ${ }^{\mathrm{d}}$ Calculated using probiotic $(\mathrm{n}=75)$, placebo $(\mathrm{n}=65)$, colonized by L. reuteri $(\mathrm{n}=13)$, and not colonized by L. reuteri $(\mathrm{n}=47)$ due to missing data.

The use of quantitative real-time PCR (qPCR) for detection of L. reuteri and E. coli enabled density-based analysis of bacterial loads. This study examined the relationships between $L$. reuteri and E. coli colonization, microbial diversity, intestinal inflammation, and crying time in a subset of infants from the Baby Biotics trial cohort. We hypothesized that infants colonized with L. reuteri would have lower E. coli colonization, greater microbial diversity, lower intestinal inflammation (calprotectin levels), and lower crying time, when compared to infants not colonized with $L$. reuteri.

\section{Results}

Sixty-five infants (31 probiotic, 34 placebo) were included in this study. Fecal samples were collected on day 28 of treatment and examined for L. reuteri and E. coli colonization, microbial diversity, and calprotectin. Some infants were excluded from analysis of diversity, calprotectin, and/or crying time due to no detection of a 16S rRNA gene PCR product or $>10 \%$ of peak profile consisting of large fragments (AluI, $\mathrm{n}=20$; Sau96I, $\mathrm{n}=10)$, insufficient sample volume $(n=7)$, or insufficient crying time data $(n=9)$, respectively.

The two groups in this study, infants colonized by L. reuteri $(\mathrm{n}=14)$ and infants not colonized by L. reuteri $(\mathrm{n}=51)$, had similar clinical characteristics (Table 1). The clinical characteristics were also representative of the original Baby Biotics trial cohort, except for higher rates of family history of allergic disease in this study.

L. reuteri and $E$. coli colonization. Of the 31 infants that received probiotic, 14 (45\%) were colonized with $L$. reuteri at day 28, whilst none of the 34 infants in the placebo group became colonized (Chi-squared test, $\mathrm{P}<0.0001)$. Of the infants colonized by L. reuteri, $93 \%(13 / 14)$ were also colonized by $E$. coli, whereas $69 \%$ (35/51) of infants not colonized by L. reuteri, were colonized by E. coli, however this difference was not statistically significant (Chi-squared test, $\mathrm{P}=0.07$ ). Infants colonized with $L$. reuteri had a median colonization density of $3.6 \times 10^{5}$ genome equivalents $/ \mathrm{ml}$ (Interquartile range $(\mathrm{IQR}): 5.0 \times 10^{4}, 8.7 \times 10^{5}$ genome equivalents $/ \mathrm{ml}$ ), while infants colonized with $E$. coli had a median colonization density of $1.5 \times 10^{7}$ genome equivalents $/ \mathrm{ml}$ (IQR: $5.0 \times 10^{6}, 3.5 \times 10^{7}$ genome equivalents $/ \mathrm{ml}$ ) (Fig. 1). There was no difference in E. coli density in infants colonized or not colonized by L. reuteri (Table 2). In infants colonized by both species, there was no association between $L$. reuteri and E. coli densities (Spearman's rank correlation coefficient, $\mathrm{r}=0.16 ; \mathrm{P}=0.60$ ).

Microbial diversity. Terminal restriction fragment length polymorphism (T-RFLP) was conducted, with peak profiles generated from FAM-labelled PCR product targeting total 16S rRNA genes using two restriction enzymes (AluI and Sau96I) and analyzed separately. Peaks were quantified to provide an estimate of intestinal microbial diversity, with a higher number of peaks representing greater diversity. There was no difference between the number of peaks in infants colonized with L. reuteri when compared to those not colonized using AluI or Sau96I (Table 2). In infants colonized by L. reuteri, there was no association between density of L. reuteri colonization and number of peaks in profiles generated by either AluI (Spearman's rank correlation coefficient, $\mathrm{r}=-0.52 ; \mathrm{P}=0.13$ ) or Sau96I digestion (Spearman's rank correlation coefficient, $\mathrm{r}=0.26 ; \mathrm{P}=0.36$ ).

Intestinal inflammation. Fecal calprotectin levels were measured to investigate inflammation within the GI tract. All 58 infants assessed had detectable levels of calprotectin (median $116.2 \mu \mathrm{g}$ of calprotectin per $\mathrm{g}$ wet feces $(\mu \mathrm{g} / \mathrm{g}), \mathrm{IQR}: 72.3,255.8)$. There was no difference in calprotectin levels between infants colonized with $L$. reuteri compared to those not colonized (Table 2). In infants colonized by L. reuteri, there was no association between density of $L$. reuteri colonization and calprotectin (Spearman's rank correlation coefficient, $\mathrm{r}=0.16 ; \mathrm{P}=0.60$ ).

Crying time. There was a reduction in infant crying over time, regardless of $L$. reuteri colonization status. In infants colonized by L. reuteri, median crying reduced from $330.0 \mathrm{~min} /$ day (IQR: $211.3,445.3 \mathrm{~min} /$ day) on day 0 to $172.5 \mathrm{~min} /$ day (IQR: $92.7,278.1 \mathrm{~min} /$ day) on day 28 (Wilcoxon signed-rank test, $\mathrm{P}=0.03$ ) (Fig. 2A). Similarly, median crying reduced from $328.4 \mathrm{~min} /$ day (IQR: $237.7,443.8 \mathrm{~min} /$ day) on day 0 to $180.0 \mathrm{~min} /$ day (IQR: 135.6 , $265.5 \mathrm{~min} /$ day) on day $28 \mathrm{in}$ infants not colonized by $L$. reuteri (Wilcoxon signed-rank test, $\mathrm{P}<0.0001$ ). At day 28 there was no difference between median infant crying time between infants colonized and infants not colonized 
A

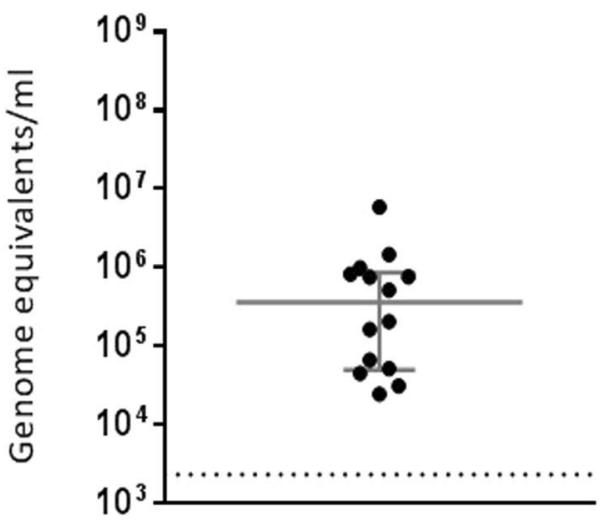

L. reuteri

B

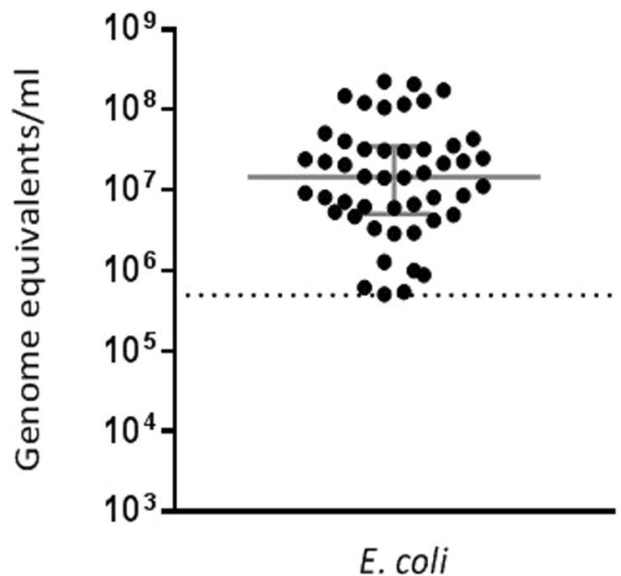

Figure 1. (A) L. reuteri $(\mathrm{n}=14)$ and $(B) E . \operatorname{coli}(\mathrm{n}=48)$ colonization levels in fecal samples collected at day 28 from infants with colic. Bars represent median \pm interquartile range; dotted lines represent assay limit of detection.

\begin{tabular}{|c|c|c|c|c|c|}
\hline \multirow[b]{2}{*}{ Outcomes } & \multicolumn{4}{|c|}{ Median (IQR) } & \multirow[b]{2}{*}{ P-value ${ }^{a}$} \\
\hline & $\mathbf{n}$ & Colonized by $L$. reuteri & $\mathbf{n}$ & Not colonized by $L$. reuteri & \\
\hline Fecal E. coli colonization (genome equivalents $/ \mathrm{ml}$ ) & 13 & $1.4 \times 10^{7}\left(3.6 \times 10^{6}, 6.9 \times 10^{7}\right)$ & 35 & $1.6 \times 10^{7}\left(5.4 \times 10^{6}, 3.6 \times 10^{7}\right)$ & 0.71 \\
\hline \multicolumn{6}{|l|}{ Fecal microbial diversity score } \\
\hline - AluI peaks & 10 & $28.5(20.5,35.8)$ & 35 & $24.0(21.0,27.0)$ & 0.14 \\
\hline - Sau96I peaks & 14 & $32.0(28.0,35.3)$ & 41 & $31.0(27.0,36.0)$ & 0.62 \\
\hline Fecal calprotectin $(\mu \mathrm{g} / \mathrm{g})$ & 13 & $135.4(89.5,568.5)$ & 45 & $114.8(67.1,237.9)$ & 0.37 \\
\hline Infant crying time (min/day) & 13 & $165.0(100.3,268.8)$ & 45 & $180.0(136.3,274.1)$ & 0.72 \\
\hline
\end{tabular}

Table 2. Outcomes by Lactobacillus reuteri colonization status, at day $28 .{ }^{a}$ Mann-Whitney U test.

by $L$. reuteri (Table 2). For the 13 infants colonized by $L$. reuteri, a positive association was found between $L$. reuteri colonization density and median infant crying time (Spearman's rank correlation coefficient, $\mathrm{r}=0.68$; $\mathrm{P}=0.01$ ) (Fig. 2B).

Secondary analyses. We additionally assessed the relationships between $E$. coli colonization and microbial diversity, intestinal inflammation and crying time (Table 3). We found no differences in microbial diversity in infants colonized or not colonized by E. coli, and no association between density of E. coli colonization and number of peaks by AluI digestion (Spearman's rank correlation coefficient, $\mathrm{r}=-0.12 ; \mathrm{P}=0.49$ ). However, in infants colonized by E. coli, there was a negative association between E. coli colonization density and number of peaks in profiles generated by Sau96I digestion (Spearman's rank correlation coefficient, $r=-0.33 ; \mathrm{P}=0.03$ ). We found no association between density of $E$. coli colonization and calprotectin (Spearman's rank correlation coefficient, $\mathrm{r}=-0.06 ; \mathrm{P}=0.69$ ). Median crying reduced from $307.5 \mathrm{~min} /$ day (IQR: $226.3,439.2 \mathrm{~min} /$ day) on day 0 to $180.0 \mathrm{~min} /$ day (IQR: $134.0,282.0 \mathrm{~min} /$ day) on day $28 \mathrm{in}$ infants colonized by $E$. coli (Wilcoxon signed-rank test, $\mathrm{P}<0.0001$ ). Similarly, median crying reduced from $352.5 \mathrm{~min} /$ day (IQR: $288.8,460.8 \mathrm{~min} /$ day) on day 0 to $168.8 \mathrm{~min} /$ day (IQR: 133.1, $220.3 \mathrm{~min} /$ day) on day 28 in infants not colonized by E. coli (Wilcoxon signed-rank test, $\mathrm{P}=0.0003$ ) (Fig. 3A). There was no statistically significant difference between median change in crying time from day 0 to day 28 between infants colonized $(81.3 \mathrm{~min} /$ day, IQR: $15.5,263.6)$ or not colonized $(193.8 \mathrm{~min} /$ day, IQR: $125.7,295.6$ ) by E. coli (Mann-Whitney U test, $\mathrm{P}=0.07$ ). For the 42 infants colonized by $E$. coli, no relationship was found between $E$. coli colonization density and median infant crying time (Spearman's rank correlation coefficient, $\mathrm{r}=0.01 ; \mathrm{P}=0.94$ ) (Fig. 3B).

\section{Discussion}

We previously reported that supplementation with L. reuteri DSM 17938 did not reduce crying time in breastfed and formula-fed infants with colic ${ }^{16}$. However, the L. reuteri colonization rates were lower than expected, potentially affecting the Baby Biotics trial results. In this substudy, we examined the relationship between L. reuteri colonization and microbiological outcomes (E. coli colonization and microbial diversity), intestinal inflammation, and crying time in a subset of infants from the trial. L. reuteri colonization rates ( $45 \%$ at $\sim 4$ months of age) in the current study were lower than those previously reported in other pediatric studies $\left(92 \%\right.$ at $\sim 2$ months $s^{11}$, 
A
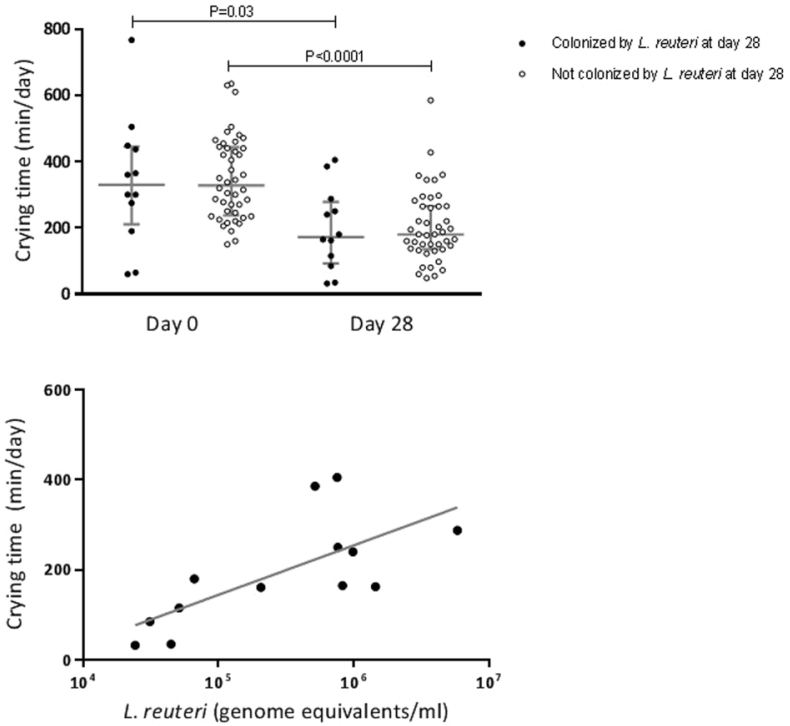

Figure 2. The association between L. reuteri colonization and infant crying time. (A) Crying time in infants colonized $(n=12)$ and not colonized $(n=44)$ by L. reuteri. Infants were categorized as colonized or not colonized by L. reuteri at both time points by L. reuteri colonization status at day 28. Bars represent median \pm interquartile range. Differences within groups (day 0 to day 28) were assessed using Wilcoxon signedrank test and differences between groups (colonized and not colonized) using Mann-Whitney U test. (B) The relationship between crying time and L. reuteri colonization density $(\mathrm{n}=13)$; Spearman's rank correlation coefficient, $\mathrm{r}=0.68 ; \mathrm{P}=0.01$.

\begin{tabular}{|l|l|l|l|l|l|l|}
\hline \multirow{2}{*}{ Outcomes } & \multicolumn{4}{|l|}{ Median (IQR) } & \multirow{2}{*}{} \\
\cline { 2 - 6 } & $\mathbf{n}$ & Colonized by E. coli & $\mathbf{n}$ & Not colonized by E. coli & P-value $^{\mathbf{a}}$ \\
\hline Fecal microbial diversity score & 36 & $25.0(21.3,29.0)$ & 9 & $22.0(16.5,26.5)$ & 0.17 \\
\hline - AluI peaks & 44 & $31.0(27.0,36.0)$ & 11 & $30.0(28.0,33.0)$ & 0.87 \\
\hline - Sau 96 I peaks & 46 & $118.0(70.2,267.3)$ & 12 & $94.2(74.4,246.5)$ & 0.58 \\
\hline Fecal calprotectin $(\mu \mathrm{g} / \mathrm{g})$ & 42 & $180.0(142.0,288.5)$ & 16 & $168.8(133.1,220.3)$ & 0.44 \\
\hline Infant crying time $(\mathrm{min} /$ day) & 42 &
\end{tabular}

Table 3. Outcomes by Escherichia coli colonization status, at day $28 .{ }^{\mathrm{a}}$ Mann-Whitney U test.

and $65 \%$ at $\sim 3$ months ${ }^{18}$ ). The reduction in colonization rates with increasing infant age suggest age at probiotic administration and/or age at sample collection, as well as the use of different microbiological detection methods (culture-dependent versus culture-independent methods in our study), may affect L. reuteri detection ${ }^{11,18}$. We found median colonization density of $L$. reuteri to be $3.6 \times 10^{5}$ genome equivalents $/ \mathrm{ml}$ in infants who were colonized with L. reuteri, which is in line with previous research that found mean L. reuteri colonization densities of $10^{4}-10^{5} \mathrm{CFU} / \mathrm{g}$ of feces after supplementation ${ }^{11,19}$. Microbial diversity was similar in infants colonized or not colonized with $L$. reuteri, consistent with Roos et al. who found that treatment with $L$. reuteri did not affect the global composition of the bacterial community ${ }^{20}$. We found no difference in calprotectin levels in infants colonized or not colonized by L. reuteri, and no association between calprotectin levels and crying time at day 28 . However, we previously reported that infants who had a reduction in crying time of at least $50 \%$ had lower calprotectin levels, suggesting gut inflammation may be implicated in infant colic, although the precise relationship remains unclear ${ }^{16}$. Crying time decreased in infants from day 0 to day 28 regardless of $L$. reuteri colonization status, in line with the natural resolution of infant colic that typically occurs when an infant reaches three to four months of age $\mathrm{e}^{21}$. There were two infants in the L. reuteri colonized group with lower crying times at day 0 than were observed in the not colonized group (approximately $60 \mathrm{~min} /$ day, versus minimum of $150 \mathrm{~min} /$ day). This may have decreased the ability to detect a reduction in crying time from day 0 to day 28 in the infants colonized with L. reuteri, however we still found a reduction in median crying time. We found no difference in crying time at day 28 between infants colonized or not colonized by L. reuteri. These findings were consistent with the original Baby Biotics trial results, and in contrast to previous reports that $L$. reuteri treatment is effective in reducing crying time in infants with colic when crying is assessed by treatment allocation ${ }^{11-15}$. These divergent findings may reflect differences in geographic location of previous studies (Italy, Poland, China and Canada) and rates of exclusive breastfeeding (majority of infants in previous studies versus 35\% in our study). Geography and method of feeding are known to impact on gut microbiota ${ }^{22-25}$, therefore our study emphasizes that results from other settings may not be generalizable to our study population, or to Australian infants in general. In infants colonized by L. reuteri, 
A

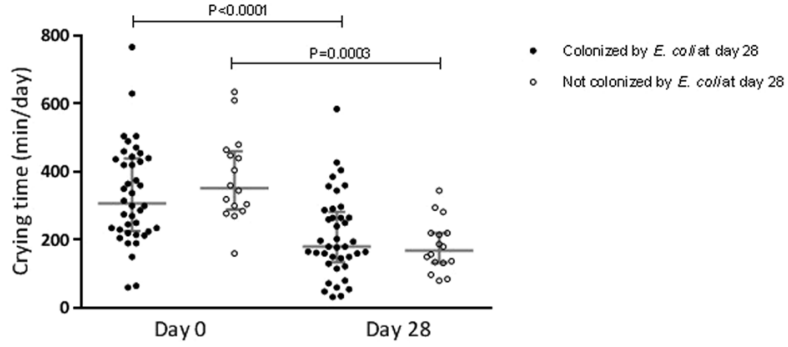

B

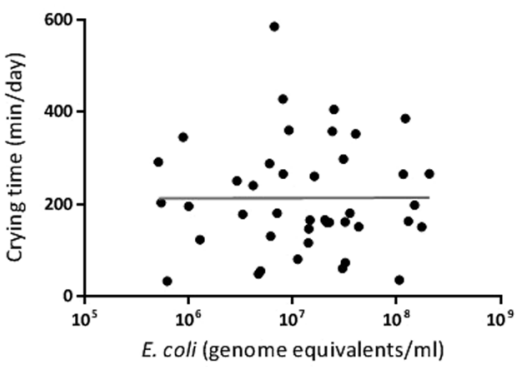

Figure 3. The association between E. coli colonization and crying time. (A) Crying time in infants colonized $(\mathrm{n}=40)$ and not colonized $(\mathrm{n}=16)$ by E. coli. Infants were categorized as colonized or not colonized by E. coli at both time points by $E$. coli colonization status at day 28. Bars represent median \pm interquartile range. Differences within groups (day 0 to day 28) were assessed using Wilcoxon signed-rank test and differences between groups (colonized or not colonized) using Mann-Whitney U test. (B) The relationship between crying time and E. coli colonization density $(n=42)$; Spearman's rank correlation coefficient, $r=0.01 ; P=0.94$.

we observed an unexpected positive relationship between $L$. reuteri density and crying time. Although this finding may generate concerns that high density of $L$. reuteri could exacerbate symptoms of colic, there does not appear to be a link with gut inflammation as there was no association between L. reuteri density and calprotectin. It is plausible that some infants may have an underlying gut environment that is associated with higher crying and also facilitates high-density $L$. reuteri growth.

In our study, we found no evidence linking E. coli to gut inflammation as measured by calprotectin. We found a negative association between $E$. coli colonization density and microbial diversity, although this was only detected with one of the enzymes used in the diversity assay. E. coli has been associated with Crohn's Disease, with decreases in microbial diversity compared with healthy participants ${ }^{26,27}$, suggesting a possible pathophysiological relationship between GI E. coli colonization and microbial diversity. Savino et al. found that infants who responded to L. reuteri treatment (a reduction of $\geq 50 \%$ crying time) had a reduction in fecal E. coli, so our finding of no difference in E. coli colonization is consistent with the outcome that treatment with L. reuteri did not reduce crying time in this study population. There was moderate evidence that infants colonized with $E$. coli had a smaller reduction in crying from baseline to day 28 , however there was no difference between crying time at day 28 when assessed by E. coli colonization status. It has been previously reported that $L$. reuteri administration was associated with a decreased presence of E. coli, suggesting a potential negative interaction between these two species ${ }^{11}$. However, we did not observe a negative relationship between L. reuteri and E. coli, and in fact, $93 \%$ of infants colonized with $L$. reuteri also had $E$. coli detected. This suggests that the findings of Savino et al. may have been due to an indirect rather than direct relationship between the two species, and/or could be another example of differences attributable to geography and feeding methods.

To our knowledge, this is the first study to investigate microbiological changes in infant colic by L. reuteri colonization status. The participants in our study are representative of help-seeking parents and carers from the general population. Because Australian pediatricians do not deliver primary care, families often present to the emergency department for common childhood concerns, especially for conditions such as colic where the burden typically occurs outside business hours. Additionally, this study included both breast and formula-fed infants and did not restrict the mother's diet to make the probiotic intervention more generalizable. This is in contrast to the majority of infant colic studies that use populations of exclusively (or predominately) breastfed infants ${ }^{11-15}$, and have mothers or infants on a cow's milk-free diet ${ }^{11,12,14}$. However, this study had several limitations. Only a subset of samples from the original trial were available for the analyses conducted in this pilot project. We performed laboratory analyses at a single time point at the end of treatment when crying time had already reduced, which may have blunted the ability to detect potential differences linked to crying time. The infant microbiome is highly variable, and it is plausible that transient changes due to probiotic colonization may have occurred earlier. Additionally, while we used robust culture-independent molecular methods to identify L. reuteri and E. coli, the qPCR primers and probes may detect other closely related species, such as the closely related Shigella spp. for the E. coli assay ${ }^{28-30}$. Nevertheless, only infants in the probiotic group had detectable L. reuteri, suggesting our assay was specific for the probiotic strain. Lastly, parents were instructed to deliver the supplement using a spoon or dropper; it is not known whether the route of administration impacted L. reuteri colonization. Advances in 
molecular methods and sequencing technology have enabled more detailed characterization of the microbiome including identification of more subtle differences in the bacterial communities present. Employing one of these methods may provide further information on differences in the GI microbiota and greater insight into potential impacts of $L$. reuteri treatment on the gut microbiome.

We found no reduction in crying time in infants colonized with $L$. reuteri and identified an unexpected positive association between $L$. reuteri density and crying time. Our results support the finding of the original Baby Biotics trial that $L$. reuteri supplementation did not reduce crying time in infants with colic in Melbourne, Australia. We also found a negative association between E. coli colonization density and microbial diversity, suggesting a possible pathophysiological relationship between E. coli colonization and microbial diversity. Our findings suggest $L$. reuteri supplementation may not be an appropriate treatment for infants with colic in some geographic settings, and that the impact of such an intervention on intestinal microbiota composition remains unclear. As our study included a small subset of infants from the Baby Biotics randomized trial, additional studies with larger sample size are required to further investigate the role of the gut microbiome in infant colic and the utility of probiotic treatment in different populations.

\section{Methods}

Study participants and design. The 65 infants included in this study were part of a previously described placebo-controlled trial in Melbourne, Australia that assessed L. reuteri supplementation as a treatment for infant colic $^{16,31}$. In brief, 167 healthy term infants aged $<13$ weeks with infant colic (modified Wessel's criteria, crying and/or fussing for $\geq 3$ hours a day, for $\geq 3$ days over seven days) were randomized to receive five drops of probiotic $\left(1 \times 10^{8}\right.$ colony forming units (CFU)/day L. reuteri DSM 17938; BioGaia AB, Stockholm, Sweden) or placebo daily for 28 days. A random sample of eight returned probiotic bottles were tested to confirm the probiotic suspension was above the required dose; mean L. reuteri recovery was $1.8 \times 10^{8} \mathrm{CFU} / 5$ drops. The trial was approved by the Human Research Ethics Committee of The Royal Children's Hospital (HREC 30111), and informed consent was obtained for all participants. 127 infants completed the original trial ( 67 probiotic, 60 placebo). For the current study, fecal samples were analyzed from the first 65 infants enrolled in the original trial who produced adequate fecal sample volume (31 probiotic, 34 placebo; Table 1). All methods were carried out in accordance with relevant guidelines and regulations. All laboratory analyses were performed blinded.

Infant crying and/or fussing time. Infant crying and/or fussing time (referred to as 'crying time') was measured using the Barr diary and analyzed as described elsewhere ${ }^{32,33}$. Infant crying and fussing times were reported by the primary caregiver and collected over a 24 hour period at baseline, and as an average of 48 hours for day 28 of the study (measured over days 28 and 29). The diaries were adjusted for missing information, with diaries that had $\geq 30 \%$ missing data excluded from analyses at the relevant time point. For diaries with $<30 \%$ missing data, mean values of crying time were generated using the equation: [episodes of daily cry/fuss bouts $]^{*}[1440 /(1440$ - minutes of missing data $)]$.

Fecal sample collection and storage. Fecal samples were collected on day 28 of the trial. Samples were collected from the infant's diaper, transferred to a sterile container, and placed immediately into the caregiver's freezer. Alternatively, the whole diaper was placed into the freezer. Frozen samples were transported on ice in an insulated container from the caregiver's freezer to the laboratory within a median of 3 days (range 0-20 days). Once received at the laboratory, diaper samples were transferred into a container. All samples were stored at $-80^{\circ} \mathrm{C}$ until use (maximum storage of 9 months from collection to DNA extraction).

Extraction of fecal genomic DNA. On the day of DNA extraction, samples were thawed and homogenized before use. Genomic DNA was extracted using the UltraClean ${ }^{\circledR}$ Fecal DNA Isolation Kit (MO BIO Laboratories, Inc., Carlsbad, USA). Briefly, $0.15 \mathrm{~g}$ of homogenized fecal sample was added to a bead/ball mixture containing $0.5 \mathrm{~g}$ of $0.1 \mathrm{~mm}$ Zirconia/Silica beads (BioSpec Products Inc., Bartlesville, USA) and four $3 \mathrm{~mm}$, undrilled, glass balls (Ajax Finechem Pty Ltd., Taren Point, Australia). DNA isolation was completed following the manufacturer's protocol from the addition of Solution S1 of the MO BIO kit. If pellets were not formed, the sample was spun in a centrifuge for an additional $3 \mathrm{~min}$ at $11,300 \mathrm{x} g$ before transferring the supernatant. DNA was eluted in a total volume of $100 \mu \mathrm{l}$.

Quantification of $L$. reuteri and E. coli. L. reuteri and E. coli were quantified by qPCR using previously published primers and probes targeting the $t u f$ and $u i d A$ genes, respectivel $y^{28-30}$. Each $25 \mu$ l reaction consisted of $2 \mu \mathrm{l}$ of genomic DNA, 1X Brilliant III Ultra-Fast QPCR Master Mix (Agilent Technologies, Santa Clara, USA), $100 \mu \mathrm{M}$ probe and either $300 \mu \mathrm{M}$ of $L$. reuteri primers or $100 \mu \mathrm{M}$ of $E$. coli primers. Assays were performed using Stratagene MX3005P qPCR instrument with an initial activation of $95^{\circ} \mathrm{C}$ for 3 min followed by 40 cycles of $95^{\circ} \mathrm{C}$ for $20 \mathrm{~s}$ and $60^{\circ} \mathrm{C}$ for $20 \mathrm{~s}$. Reactions were performed in duplicate and the mean cycle threshold (Ct) value calculated. Genomic DNA from L. reuteri DSM 17938 and E. coli ATCC 25922 were used for standard curves; a mean Ct of $<35$ and $<34$, respectively, were considered positive. The assay detection limits were determined by validation against a panel of related bacteria, and no template controls. Samples with a higher $\mathrm{Ct}$ than an extraction control were considered negative. Bacterial density data are reported as genome equivalents $/ \mathrm{ml}$ (assuming one copy of the target gene per genome, one genome per CFU, and a genome size of $2.0 \mathrm{Mb}$ for L. reuteri and $5.24 \mathrm{Mb}$ for E. coli).

Microbial diversity. Microbial diversity was examined using T-RFLP, using a modified version of a method previously described ${ }^{34,35}$. Briefly, the $16 \mathrm{~S}$ rRNA gene was amplified using universal primers FAM27f and 519r 
(Sigma-Aldrich, Castle Hill, Australia) and the HotStarTaq ${ }^{\circledR}$ kit (Qiagen). Each $25 \mu$ l reaction contained $5 \mu \mathrm{l}$ genomic DNA, 1.25 U DNA Polymerase, 1 X PCR Buffer, $2.5 \mathrm{mM} \mathrm{MgCl}_{2}, 200 \mu \mathrm{M}$ dNTPs, $500 \mathrm{nM}$ fluorescently labelled forward primer and $500 \mathrm{nM}$ reverse primer. PCR cycling conditions were denaturation at $95^{\circ} \mathrm{C}$ for $5 \mathrm{~min}$, followed by 25 cycles of $94^{\circ} \mathrm{C}$ for $15 \mathrm{~s}, 50^{\circ} \mathrm{C}$ for $15 \mathrm{~s}, 72^{\circ} \mathrm{C}$ for $1 \mathrm{~min}$, and an extension of $50^{\circ} \mathrm{C}$ for $1 \mathrm{~min} 30 \mathrm{~s}$ and $72^{\circ} \mathrm{C}$ for $6 \mathrm{~min}$. Triplicate reactions were pooled. Samples with a PCR product of $480-540$ base pairs were used for further processing. PCR products were purified using a Wizard SV Gel and PCR Clean-Up System column (Promega, Annandale, Australia). T-RFLP fragments were generated using separate reactions for enzymes AluI and Sau96I (New England Biolabs, Ipswich, USA). In $25 \mu$ l reactions, approximately 50-100 ng of purified PCR product was digested with $5 \mathrm{U}$ of enzyme and 1 X NEBuffer 4 (New England Biolabs) in a water bath $\left(37^{\circ} \mathrm{C}\right.$ overnight). PCR products were precipitated and analysed by an AB3730 DNA analyser using AB GeneMapper software (Applied Biosystems, Carlsbad, USA) at the Australian Genome Research Facility, Parkville, Australia. Separate peak profiles were generated for each enzyme. Analysis of T-RFLP peak profiles was performed as described previously ${ }^{34}$.

Calprotectin. $\quad 100 \mathrm{mg}$ of fecal sample and $4.9 \mathrm{ml}$ of Extraction Buffer were mixed for $3 \mathrm{~min}$ in the Fecal Extraction Device (CALPRO AS, Lysaker, Norway) on a Ratek MPS1 plate shaker. Fecal extracts were stored at $4{ }^{\circ} \mathrm{C}$ and used within 5 days. Calprotectin levels were measured by enzyme linked immunosorbent assay $\left(\right.$ CALPROLAB $^{\mathrm{TM}}$, CALPRO AS) according to manufacturer's protocol. The optical density was measured at $405 \mathrm{~nm}$ using an ELx808 ${ }^{\mathrm{TM}}$ Absorbance Microplate Reader (BioTek) and the KCjunior ${ }^{\mathrm{TM}}$ (BioTek) package to determine calprotectin levels.

Statistical analyses. Analyses were completed using GraphPad Prism version 7.01 for Windows (GraphPad Software, San Diego California USA). The measures of E. coli colonization, microbial diversity, intestinal inflammation, and crying time were stratified by L. reuteri colonization status and tested for normality using the D'Agostino-Pearson K-squared test. As the majority of data sets were not normally distributed, non-parametric tests were used throughout. Differences between categorical variables were assessed using the Chi-Squared test. Differences within groups (colonized or not colonized by L. reuteri; colonized or not colonized by E. coli) were assessed using the Wilcoxon signed-rank test, and between groups using the Mann-Whitney U test. Correlations were assessed using Spearman's rank correlation coefficient. All tests were two-tailed and $\mathrm{P}<0.05$ was considered statistically significant.

Data availability. The datasets generated during and/or analysed during the current study are available from the corresponding author on reasonable request.

\section{References}

1. Lucassen, P. L. et al. Systematic review of the occurrence of infantile colic in the community. Arch. Dis. Childh., Lond. 84, 398-403 (2001).

2. Sung, V. et al. Probiotics to prevent or treat excessive infant crying: systematic review and meta-analysis. JAMA Pediatr. 167, $1150-1157$ (2013).

3. Garrison, M. M. \& Christakis, D. A. A systematic review of treatments for infant colic. Pediatrics 106, 184-190 (2000).

4. Savino, F. et al. Molecular identification of coliform bacteria from colicky breastfed infants. Acta Paediatr. 98, 1582-1588 (2009).

5. Savino, F. et al. Intestinal microflora in breastfed colicky and non-colicky infants. Acta Paediatr. 93, 825-829 (2004).

6. Savino, F. et al. Antagonistic effect of Lactobacillus strains against gas-producing coliforms isolated from colicky infants. BMC Microbiol. 11, 157 (2011).

7. Savino, F. et al. Comparison of formula-fed infants with and without colic revealed significant differences in total bacteria, Enterobacteriaceae and faecal ammonia. Acta Paediatr. 106, 573-578 (2017).

8. Mentula, S., Tuure, T., Koskenala, R., Korpela, R. \& Kononen, E. Microbial composition and fecal fermentation end products from colicky infants- a probiotic supplementation pilot. Micro. Ecol. Health Dis. 20, 37-47 (2008).

9. Rhoads, J. M. et al. Altered fecal microflora and increased fecal calprotectin in infants with colic. J. Pediatr. 155, 823-828 (2009).

10. Olafsdottir, E., Aksnes, L., Fluge, G. \& Berstad, A. Faecal calprotectin levels in infants with infantile colic, healthy infants, children with inflammatory bowel disease, children with recurrent abdominal pain and healthy children. Acta Paediatr. 91, 45-50 (2002).

11. Savino, F. et al. Lactobacillus reuteri DSM 17938 in infantile colic: a randomized, double-blind, placebo-controlled trial. Pediatrics 126, 526-533 (2010).

12. Savino, F., Pelle, E., Palumeri, E., Oggero, R. \& Miniero, R. Lactobacillus reuteri (American Type Culture Collection Strain 55730) versus simethicone in the treatment of infantile colic: a prospective randomized study. Pediatrics 119, 124-130 (2007).

13. Szajewska, H., Gyrczuk, E. \& Horvath, A. Lactobacillus reuteri DSM 17938 for the Management of Infantile Colic in Breastfed Infants: A Randomized, Double-Blind, Placebo-Controlled Trial. J. Pediatr. 162, 257-262 (2013).

14. Mi, G. L. et al. Effectiveness of Lactobacillus reuteri in infantile colic and colicky induced maternal depression: a prospective single blind randomized trial. Antonie van Leeuwenhoek 107, 1547-1553 (2015).

15. Chau, K. et al. Probiotics for infantile colic: a randomized, double-blind, placebo-controlled trial investigating Lactobacillus reuteri DSM 17938. J. Pediatr. 166, 74-78 (2015).

16. Sung, V. et al. Treating infant colic with the probiotic Lactobacillus reuteri: double blind, placebo controlled randomised trial. BMJ 348, g2107 (2014).

17. de Weerth, C., Fuentes, S., Puylaert, P. \& de Vos, W. M. Intestinal microbiota of infants with colic: development and specific signatures. Pediatrics 131, e550-558 (2013).

18. Abrahamsson, T. R., Sinkiewicz, G., Jakobsson, T., Fredrikson, M. \& Bjorksten, B. Probiotic lactobacilli in breast milk and infant stool in relation to oral intake during the first year of life. J. Pediatr. Gastroenterol. Nutr. 49, 349-354 (2009).

19. Shornikova, A. V., Casas, I. A., Isolauri, E., Mykkanen, H. \& Vesikari, T. Lactobacillus reuteri as a therapeutic agent in acute diarrhea in young children. J. Pediatr. Gastroenterol. Nutr. 24, 399-404 (1997).

20. Roos, S. et al. 454 pyrosequencing analysis on faecal samples from a randomized DBPC trial of colicky infants treated with Lactobacillus reuteri DSM 17938. PloS one 8, e56710 (2013).

21. Illingworth, R. S. Three-months' colic. Arch. Dis. Childh., Lond. 29, 165-174 (1954).

22. Fallani, M. et al. Intestinal microbiota of 6-week-old infants across Europe: geographic influence beyond delivery mode, breastfeeding, and antibiotics. J. Pediatr. Gastroenterol. Nutr. 51, 77-84 (2010). 
23. Prideaux, L. et al. Impact of ethnicity, geography, and disease on the microbiota in health and inflammatory bowel disease. Inflamm. Bowel Dis. 19, 2906-2918 (2013).

24. Suzuki, T. A. \& Worobey, M. Geographical variation of human gut microbial composition. Biol. Lett. 10, 20131037 (2014).

25. Yatsunenko, T. et al. Human gut microbiome viewed across age and geography. Nature 486, 222-227 (2012).

26. Willing, B. et al. Twin studies reveal specific imbalances in the mucosa-associated microbiota of patients with ileal Crohn's disease. Inflamm. Bowel Dis. 15, 653-660 (2009).

27. Willing, B. P. et al. A pyrosequencing study in twins shows that gastrointestinal microbial profiles vary with inflammatory bowel disease phenotypes. Gastroenterology 139, 1844-1854e1 (2010).

28. Million, M. et al. Obesity-associated gut microbiota is enriched in Lactobacillus reuteri and depleted in Bifidobacterium animalis and Methanobrevibacter smithii. Int. J. Obes. (Lond.) 36, 817-825 (2012).

29. Chern, E. C., Siefring, S., Paar, J., Doolittle, M. \& Haugland, R. A. Comparison of quantitative PCR assays for Escherichia coli targeting ribosomal RNA and single copy genes. Lett. Appl. Microbiol. 52, 298-306 (2011).

30. Chern, E. C., Brenner, K. P., Wymer, L. \& Haugland, R. A. Comparison of Fecal Indicator Bacteria Densities in Marine Recreational Waters by QPCR. Water Qual. Expo. Health 1, 203-214 (2009).

31. Sung, V. et al. Probiotics to improve outcomes of colic in the community: Protocol for the Baby Biotics randomised controlled trial. BMC Pediatr. 12, 135 (2012)

32. Barr, R. G., Kramer, M. S., Pless, I. B., Boisjoly, C. \& Leduc, D. Feeding and temperament as determinants of early infant crying/ fussing behavior. Pediatrics 84, 514-521 (1989).

33. Barr, R. G., Kramer, M. S., Boisjoly, C., McVey-White, L. \& Pless, I. B. Parental diary of infant cry and fuss behaviour. Arch. Dis. Childh., Lond. 63, 380-387 (1988).

34. Ismail, I. H. et al. Reduced gut microbial diversity in early life is associated with later development of eczema but not atopy in highrisk infants. Pediatr. Allergy Immunol. 23, 674-681 (2012).

35. Osborne, C. A., Rees, G. N., Bernstein, Y. \& Janssen, P. H. New threshold and confidence estimates for terminal restriction fragment length polymorphism analysis of complex bacterial communities. Appl. Environ. Microbiol. 72, 1270-1278 (2006).

\section{Acknowledgements}

We thank all study participants and families who took part in the Baby Biotics trial, Melissa Wake and Harriet Hiscock for their contribution into the design of the Baby Biotics trial, Frances Oppedisano, Susie Germano and Anne Balloch for assistance with laboratory testing, Elissa York for patient recruitment, and CALPRO for providing Fecal Extraction Devices and Calprotectin ELISAs.

\section{Author Contributions}

M.N. developed and performed laboratory assays, completed statistical analysis and drafted the manuscript. M.T. designed and oversaw the study, advised on laboratory outcome measures and interpretation, and oversaw manuscript development. C.S. and E.D. oversaw laboratory assays and their interpretation, and assisted with manuscript development. FM provided statistical advice. S.J. completed the analysis of terminal restriction fragment polymorphism peak profiles. V.S. designed the original Baby Biotics trial with M.T. and F.M. All authors read and approved the final manuscript.

\section{Additional Information}

Competing Interests: The Baby Biotics clinical trial was supported by the Georgina Menzies Maconachie Charitable Trust administered by Equity Trustees. VS (Postgraduate Scholarship 607447) and FM (ECF: 1037449, CDF: 1111160) have support from the Australian National Health and Medical Research Council (NHMRC) for the submitted work. VS is supported by a NHMRC ECF (1125687), a Royal Australasian College of Physicians Cottrell Research Establishment Fellowship, and has been supported by a Melbourne Children's Clinician Scientist Fellowship. CS holds an Australian NHMRC CDF: 1087957. BioGaia Sweden supplied the investigational product and placebo at no cost. Calpro AS supplied CalproLab ELISA kits for the analysis of calprotectin levels at no cost. BioGaia, Calpro AS, and Equitee Trustees were independent of the trial and its researchers and played no role in the trial design; in the collection, analysis, and interpretation of data; in the writing of the report; and in the decision to submit the article for publication. MT is an inventor on a patent owned by the Murdoch Children's Research Institute "A method of inducing tolerance", and is an employee of and holds share interest in ProTA. MT is a member of medical advisory board for Nestle Nutrition Institute, past member of Global Scientific Advisory Board for Danone Nutricia, received honoraria for presentations at symposia sponsored by Danone Nutricia, received probiotic and placebo research products from Nestlé Research Centre Switzerland and Dicofarm Italy for studies unrelated to this trial. CS and ED received probiotic strains from Blis Technologies for studies unrelated to this trial. Murdoch Children's Research Institute is supported by the Victorian Government's Operational Infrastructure Support Program. The other authors have indicated they have no financial relationships relevant to this article to disclose.

Publisher's note: Springer Nature remains neutral with regard to jurisdictional claims in published maps and institutional affiliations.

Open Access This article is licensed under a Creative Commons Attribution 4.0 International License, which permits use, sharing, adaptation, distribution and reproduction in any medium or format, as long as you give appropriate credit to the original author(s) and the source, provide a link to the Creative Commons license, and indicate if changes were made. The images or other third party material in this article are included in the article's Creative Commons license, unless indicated otherwise in a credit line to the material. If material is not included in the article's Creative Commons license and your intended use is not permitted by statutory regulation or exceeds the permitted use, you will need to obtain permission directly from the copyright holder. To view a copy of this license, visit http://creativecommons.org/licenses/by/4.0/.

(c) The Author(s) 2017 